\title{
Lithium and boron burning $S(E)$-factor measurements at astro- physical energies via the Trojan Horse Method
}

L. Lamia ${ }^{1, a}$, C. Spitaleri ${ }^{1,2}$, R. G. Pizzone ${ }^{2}$, S. Cherubini ${ }^{1,2}$, S. Degl'Innocenti ${ }^{3,4}$, J. Grineviciute ${ }^{1}$, M. Gulino ${ }^{1,5}$, M. La Cognata ${ }^{1}$, A. Mukhamedzhanov ${ }^{6}$, S. Palmerini ${ }^{1}$, L. Pappalardo ${ }^{7}$, P.G. Prada Moroni $^{3,4}$, S.M.R. Puglia ${ }^{1,2}$, G.G. Rapisarda ${ }^{1,2}$, S. Romano ${ }^{1,2}$, M.L. Sergi ${ }^{1,2}$, E. Tognelli ${ }^{3,4}$, and A. Tumino 2,5

${ }^{1}$ Dipartimento di Fisica e Astronomia, Università di Catania, Italy

${ }^{2}$ Laboratori Nazionali del Sud, INFN-LNS, Catania, Italy

${ }^{3}$ Dipartimento di Fisica, Università di Pisa, Italy

${ }^{4}$ INFN Sezione di Pisa, Italy

${ }^{5}$ Università degli Studi di Enna "Kore", Italy

${ }^{6}$ Cyclotron Institute, Texas A\&M University, College Station, Texas

${ }^{7}$ Dipartimento di Fisica e Scienze della Terra, Università di Ferrara, Italy

\begin{abstract}
The residual amount of light elements lithium, beryllium and boron ( $\mathrm{LiBeB})$ abundances in stellar atmospheres has been largely accepted as one of the most powerful probes for understanding stellar structure and mixing phenomena. They are in fact gradually destroyed at different depths of stellar interior mainly by (p, $\alpha$ ), thus their fate in stars is an incomparable tool for studying mixing processes. In order to avoid extrapolation procedures on the available direct $\mathrm{S}(\mathrm{E})$-factor measurements, the Trojan Horse Method (THM) has been developed, allowing one to measure the bare nucleus S(E)factor for astrophysically relevant reactions without experiencing Coulomb penetrability effects. Here, a summary on the recent ${ }^{6,7} \mathrm{Li}$ and ${ }^{11} \mathrm{~B} \mathrm{TH}$ investigations will be given and the corresponding results discussed.
\end{abstract}

\section{Introduction}

Stellar structure and mixing mechanisms for low-mass Main-Sequence stars have been a matter of study for a long time, with particular regard to the understanding of surface abundances. The "depletion problem" of light elements lithium, beryllium and boron $(\mathrm{LiBeB})$ has triggered a large amount of work since they have been considered as one of the most important and powerful probes for understanding young F-G stellar interior ([1]). For such stars, Standard Stellar Models do not take into account the possibility of "communication" between the convective zone and the nuclear destruction zone where the burning of such elements occurs mainly via $(\mathrm{p}, \alpha)$ reactions induced at temperatures of about $\mathrm{T}_{6} \sim 10$. This means that the residual $\mathrm{LiBeB}$ abundances in such stars would have to reflect the abundances of the original interstellar gas. In contrast, the observational evidence suggests that for F main sequence young stars there is a depletion of lithium and beryllium as the observations on

\footnotetext{
ae-mail: llamia@lns.infn.it
} 
Hyades, Praesepe ( $\sim 600 \mathrm{My})$ and on other young clusters reveal, while there is no evidence of this depletion in F pre-main sequence stars according to the observations of the Pleiades cluster ( $70 \mathrm{My})$. This evidence is interpreted as signature of non-standard mixing mechanisms acting inside these stars: in particular, slow-mixing mechanisms induced by stellar rotations seem to give the largest contribution for the description of the current observational status (see for details [1]). In addition, the still unsolved lithium observational scenario, as recently highlighted by the studies of [2] and [3], makes its understanding an exciting challenge.

The understanding of the framework mentioned above depends upon the improvement of both observational techniques and theoretical models. For this reason, low-energy bare-nucleus cross sections of LiBeB-burning reactions must be known precisely since they are one of the key physical inputs for stellar evolutionary code. However, direct measurements of nuclear reactions at astrophysical energies are usually difficult to perform. In such situations the corresponding Gamow window lies at energies at which the cross-section values decrease exponentially to the nano-or-picobarn regime because of the Coulomb barrier between the interacting nuclei ([4]). Thus the energy region of interest for astrophysics is usually explored by means of extrapolation procedures on the bare-nucleus $\mathrm{S}(\mathrm{E})_{b}$-factor because it smoothly varies with the energy, thus removing the exponential drop of the cross section ([4]).

In order to avoid extrapolation procedures on low-energy direct data, strongly affected by electron screening effects, the application of the Trojan Horse Method (THM) ([5-7]) allows one to measure the bare nucleus $\mathrm{S}(\mathrm{E})_{b}$-factor for charged-particle induced reactions at astrophysical energies without experiencing both Coulomb penetrability and electron screening effects (see $[8,9]$ ). In addition, thanks to the pole-invariance effects, it allows for the study of the same binary reaction of interest for astrophysics by using different Trojan Horse (TH) nuclei, as explained in [10]. However, it must be stressed here that the $\mathrm{TH} \mathrm{S}(\mathrm{E})_{b}$-factor determination requires a normalization procedure to the available direct measurements performed at higher energies ([7]), thus making the TH as a complementary experimental technique for nuclear astrophysics aimed to reach the ultra-low energy region of interest for astrophysical applications. It has been used to shed light on different astrophysical problems, such as the study of light element burning reactions [11-20], CNO reactions [21-25], and removing/producing neutron reactions [26-28].

\section{The ${ }^{11} \mathbf{B}\left(\mathbf{p}, \alpha_{0}\right)^{8} \mathrm{Be}$ reaction: main results}

The boron burning reaction ${ }^{11} \mathrm{~B}\left(\mathrm{p}, \alpha_{0}\right)^{8} \mathrm{Be}$ has been investigated by means of the THM application to the quasi-free ${ }^{2} \mathrm{H}\left({ }^{11} \mathrm{~B}, \alpha_{0}{ }^{8} \mathrm{Be}\right) \mathrm{n}$ reaction (see [29] for details), by using the deuteron as a typical THnucleus due to its simple p-n structure and to its relatively low $(\sim 2.25 \mathrm{MeV})$ binding energy. In this framework, the proton will act as participant while the neutron as spectator of the virtual two-body reaction of astrophysical interest [29]. To this end, a devoted experiment has been performed at LNS of Catania, by using a $27 \mathrm{MeV}{ }^{11} \mathrm{~B}$ beam delivered onto a $\sim 150 \mu \mathrm{g}$ thick $\mathrm{CD}_{2}$ target. The produced $\alpha$ particles were detected by means of standard position sensitive silicon detectors (PSD). No detection was necessary for the exiting neutrons, since their kinematical quantities are determined once the angles and energies of $\alpha$ and ${ }^{8} \mathrm{Be}$ have been measured. The ${ }^{8} \mathrm{Be}$ events have been reconstructed as a "coincidence-event" in a DPSD (Dual PSD) detector, basically designed as two standard PSD detectors mounted one-above-the-other and separated by a $\sim 1 \mathrm{~cm}$ of empty space. The experimental setup has been then placed in the CAMERA 2000 scattering chamber in order to cover the QF-angular region, i.e. the angular region in which a strong (even dominant) contribution of the quasi-free mechanism is expected by kinematical calculations. The selection of the quasi-free mechanism, representing one of the most crucial steps of a typical THM analysis, has been performed by studying the experimental momentum distribution for the $\mathrm{p}$-n relative motion inside the deuteron and its distortion from 
simple PWIA approximation have been evaluated by performing DWBA calculations by means of the FRESCO code. In addition, the influence of the d-state deuteron wave function component on the THM data have been studied in [30], giving negligible effects. After selection of the QF-contribution, the $\mathrm{S}(\mathrm{E})$-factor was extracted and reported on the left after normalization to the available direct data. The extracted value of $\mathrm{S}(0) \mathrm{THM}=2.07 \pm 0.41 \mathrm{MeV} b$ is in good agreement with the extrapolated one. It should be noted that the value of $\mathrm{S}(0)$ for the ${ }^{11} \mathrm{~B}(\mathrm{p}, \alpha)^{8} \mathrm{Be}$ reaction is dominated by the $\alpha_{1}$ contribution, with the $\alpha_{0}$ part being $\sim 1 \%$ of the total value. The value of the electron screening potential parameter $\left(\mathrm{U}_{e}\right) \mathrm{THM}=472 \pm 160 \mathrm{eV}$ is in agreement within the experimental errors with the value of $\mathrm{Ue}=430 \pm 80 \mathrm{eV}$ reported in the NACRE compilation [31].

\section{The ${ }^{6,7} \mathrm{Li}(\mathbf{p}, \alpha)^{3,4} \mathrm{He}$ reaction: main results}

The main burning channels for the lithium isotopes have been measured recently by means of the THM. It has been necessary because of the recent measurements of $[32,33]$ in which more precise direct measurements of the $\mathrm{S}(\mathrm{E})$-factor (i.e. total error bars of about $5 \%$ on average) have been performed in the energy range of interest for astrophysics. These data have been used as new normalization points together with the other experimental data provided by the NACRE compilation [31]. For the ${ }^{6} \mathrm{Li}$ case, the THM analysis of [34] gives $\mathrm{S}(0)=3.44 \pm 0.39 \mathrm{MeV}$ barns and $\mathrm{U}_{e}=355 \pm 100$ $\mathrm{eV}$. The THM reaction rate deviates from $\sim 5 \%$ to $\sim 15 \%$ as the temperature decreases from 1 down to $10^{-2} \mathrm{~T}_{9}$, if compared with the evaluation of [35] (left panel of Fig.1). To evaluate the astrophysical implications of these results, the FRANEC code has been used for pre-main sequence (PMS) models since the surface ${ }^{6} \mathrm{Li}$ is essentially burned in this stellar phase [36]. From such calculations, a clear influence of the temperature at the bottom of the convective envelope on the final amount of lithium has been found, i.e., the higher the metallicity, and/or the lower the stellar mass, the deeper and hotter the base of the convective envelope. For the ${ }^{7} \mathrm{Li}$ case [37], the THM measurement returns $\mathrm{S}(0)=53 \pm 5$ kev $\mathrm{b}$ and $\mathrm{U}_{e}=425 \pm 60 \mathrm{eV}$ for the bare-nucleus $\mathrm{S}(\mathrm{E})$-factor at zero energy and the electron screening potential, respectively. The reaction rate determination shows that the THM reaction rate deviates from $\sim 5 \%$ to $\sim 13 \%$ as the temperature decreases from $\mathrm{T}_{9}=1$ down to $\mathrm{T}_{9}=10^{-2}$ (right panel of Fig.1). Its astrophysical impact has been evaluated in red-giant (RGB) star's framework.

Acknowledgments. This work has been partially supported by the Italian Ministry of the University under Grant RBFR082838 (FIRB 2008).

\section{References}

[1] Boesgaard A M The light elements lithium, beryllium and boron (A. Mc William, Vol.4, 2004)

[2] Lind K et al., A\&A 554, A96 (2013)

[3] Caffau, E., Nature, 477, Issue 7362, pp. 67-69 (2011)

[4] Rolfs, C. \& Rodney, W., Cauldrons in the Cosmos (The Univ. of Chicago press, Chicago, 1988)

[5] Baur G et al., Phys.Lett. B, 178, 135 (1986)

[6] Spitaleri C et al., Phys.Rev.C, 60, 055802 (1999)

[7] Spitaleri C et al., Phys.Rev.C, 69, 055806 (2004)

[8] Tumino, A., et al., Phys.Rev.Lett., 98, 252502 (2007)

[9] Tumino, A., et al. , Phys. Rev. C, 78, 064001 (2008)

[10] Pizzone, R. G., et al., Phys. Rev. C, 83, 04580 (2011)

[11] Pizzone, R.G. et al. , Phys. Rev. C, 71, 058801 (2005) 

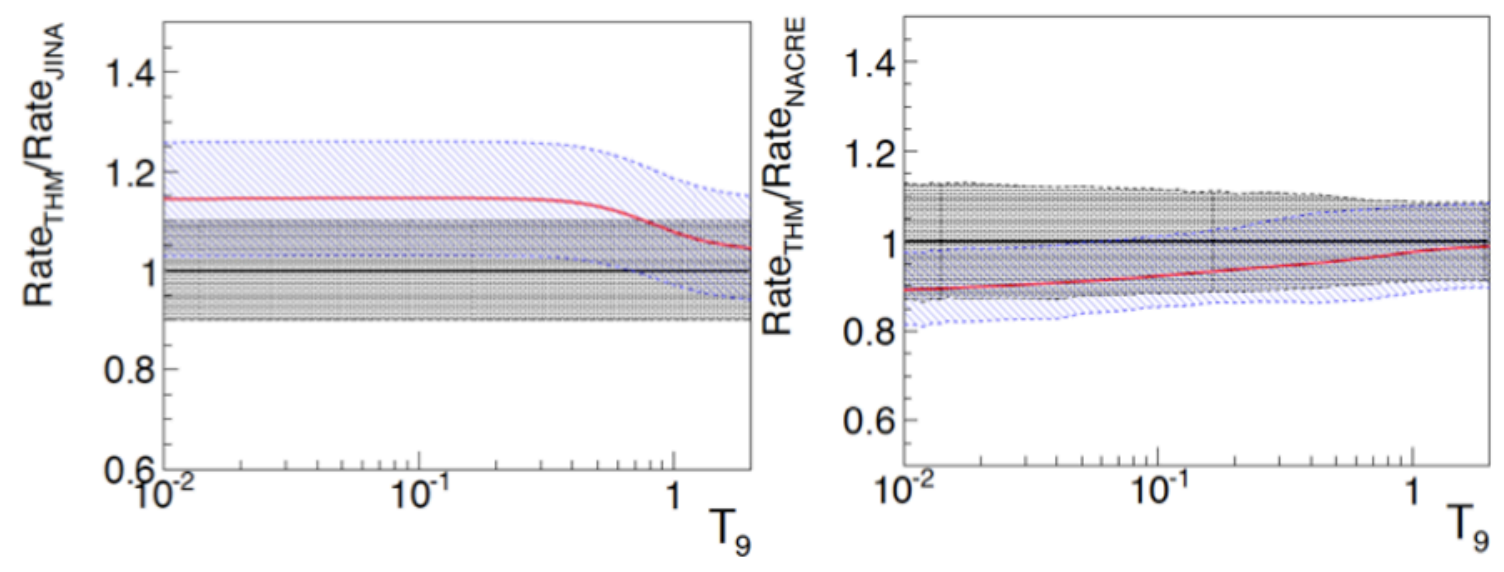

Figure 1. Left panel Ratio between the ${ }^{6} \mathrm{Li}(\mathrm{p}, \alpha)$ THM reaction rate and the JINA REACLIB [35] one (red line), together with the corresponding uncertainties (dashed area). Right panel Ratio between the ${ }^{7} \operatorname{Li}(\mathrm{p}, \alpha) \mathrm{THM}$ reaction rate and the NACRE one (red line), together with the corresponding uncertainties (dashed area).

[12] Tumino, A., et al., Eur. Phys. J., A 27, s01, 243-248 (2006)

[13] Romano, S., et al., Eur. Phys. J., A 27, s01, 221-225 (2006)

[14] Pizzone, R. G. et al., A\&A, 398, 423 (2003)

[15] Pizzone, R.G. et al., A\&A. 438, 779 (2005)

[16] Lamia, L., et al., Nucl. Phys. A, 787, 309c (2007)

[17] Lamia, L., et al., Nuovo Cimento della Società Italiana di Fisica-C, 31, 423 (2008)

[18] Tumino, A., et al., Phys.Lett. B, 700, 111-115 (2011)

[19] Tumino, A., et al., Phys.Lett. B, 705, 546 (2011)

[20] Pizzone R.G. et al., Phys. Rev. C, 87, 025805 (2013)

[21] La Cognata, M., et al., Eur.Phys.J., 27, Issue 1, 249-254 (2006)

[22] Sergi, M.L., et al., Phys.Rev.C, 82, 032801(R) (2010)

[23] La Cognata, M., et al., Astr. Phys. Journ., 708, 796 (2010)

[24] La Cognata, M., et al., Astr. Phys. Lett., 739, L54 (2011)

[25] Palmerini S. et al., The Astrophysical Journal, 764, 128 (2013)

[26] Tumino, A., et al., Eur. Phys. J., A 25, s01, 649-650 (2005)

[27] Gulino, M. et al., J. Phys. G, 37, 125105 (2010)

[28] La Cognata, M., et al. 2012, Phys.Rev.Lett., 109, 232701 (2012)

[29] Lamia, L., et al., J. Phys. G, 39, 015106 (2012)

[30] Lamia, L. et al., Phys. Rev. C, 85, 025805 (2012)

[31] Angulo, C. et al., Nucl. Phys. A, 656, 3 (1999)

[32] Cruz J. et al., Phys. Lett. B, 624, 181 (2005)

[33] Cruz J et al., Journ. of Phys. G, 35, 014004 (2008)

[34] Lamia, L. et al., The Astrophysical Journal, 768, 65, (2013)

[35] Cyburt, R. H., Amthor, A. M., Ferguson, R., et al., The Astroph. Journ., 189, 240 (2010)

[36] Tognelli, E., et al. , A\&A, 533, A109 (2011)

[37] Lamia, L. et al., Astron. \& Astrophys., 541, A158 (2012) 